\title{
On the spacecraft structure model validation and test philosophy
}

\author{
Guglielmo S. Aglietti ${ }^{1}$, Marcello Remedia ${ }^{2}$ \\ University of Surrey, Guildford, GU2 7XH, UK \\ Matteo Appolloni ${ }^{3}$ \\ European Space Agency (ESA), ESTEC, Netherlands \\ Andrew Kiley ${ }^{4}$ \\ Airbus Defence \& Space, Stevenage, SG1 2AS, UK
}

\begin{abstract}
This article examines the new practice of Virtual Shaker Testing (VST), starting from its motivation to its practical implementations and future possible implications. The issues currently experienced during large satellites' vibration testing are discussed, examining practical examples that highlight the coupling existing between the item under test and facility, and that are the basis for the motivation behind the new methodology (i.e. VST). VST is proposed as a way to bypass some of these issues, and here its use as a pre and post shaker test tool is discussed. In the article VST is applied to real test cases (Airbus' large spacecraft Bepi Colombo, built for the European Space Agency's first mission to Mercury), showing computations and real physical test data to illustrate the advantages of the methodology. These are mostly in terms of de-risking of the physical test campaigns (due to the capability to simulate realistically the future physical test thus reducing the probability of aborts and stops during the runs), and an improvement of the quality of the correlation process and related FEM update (resulting from the capability to separate the dynamics of the satellite from the effects of the test equipment); ultimately providing a tool to address questions arising from test response observations, which are many. This tool also offers the possibility to improve vibration testing using 6 DOF facilities. The article is concluded articulating a possible way forward to take maximum advantage of the new methodology, drawing a parallel with the current Satellite/Launch Vehicle Coupled Load Analysis cycles, and proposing a different design and validation philosophy.
\end{abstract}

\footnotetext{
${ }^{1}$ Professor, g.aglietti@ surrey.ac.uk

${ }^{2}$ Research Fellow, m.remedia@ surrey.ac.uk

${ }^{3} \mathrm{MSc}$, matteo.appolloni@esa.int

${ }^{4} \mathrm{Mr}$, andy.kiley@airbus.com
} 


\section{Background}

The main methodology currently available to ensure that a spacecraft can successfully withstand the launch environment is based on computations of the launch loads on the spacecraft, performed utilizing a mathematical model that includes launch vehicle and its payload (i.e. the spacecraft/satellite). The two are mathematically coupled together into a single mechanical model, subjected to loads that represent the effect of propulsion and other forces acting on the launch vehicle during its ascent. The accuracy of these satellite/launch vehicle Coupled Load Analyses (CLA) is a cornerstone for the structural verification process.

However, as to start the design of the satellite structure it is necessary to have some idea of the launch loads, the starting point are the "generic" requirements and specifications contained in the Launch Vehicle Manual (Quasi Static Loads, minimum natural frequencies, sinusoidal load environment, etc.). Later on during the design process, as a Finite Element Model (FEM) of the Satellite becomes available, it is possible to perform one or more satellite/launch vehicle CLAs to refine the predictions of the launch loads. The number of CLA cycles performed is mainly decided by the satellite prime authority. For European launchers the process typically centres on some preliminary satellite/launch vehicle CLAs, which can be performed initially on a non-validated satellite mathematical model by launcher interface authority, and later by the same authority on a validated satellite FEM. The final satellite/launch vehicle CLA is generally performed by the launcher prime contractor, and clearly this 'final' CLA needs to be based upon a level of confidence that must come from a validated satellite FEM.

The results of the CLA are clearly specific for the satellite being analysed and therefore are deemed more accurate predictions of the responses that what would be obtained by simply applying to a satellite the generic structural requirements stated in the launcher manual. Therefore, based on CLA predictions, the satellite prime typically asks for deviations of the test inputs from the test specs described in the launcher manual (typically in the form of notches on the curves that represent the sinusoidal loads) as blind application of the specifications, e.g. without considering spacecraft resonances, would produce significant and non-realistic over-tests.

Indeed this is all based on the assumption that the FEM gives an accurate representation of the real dynamics of the physical system/spacecraft, and therefore it is necessary to carry out an initial correlation activity to compare FEM predicted responses with real physical responses retrieved during hardware tests.

Various techniques and algorithms have been developed to carry out FEM numerical-experimental correlation, but vector based techniques are still the most commonly used: examples are Modal Assurance Criterion (MAC) [1], 
Response Vector Assurance Criterion (RVAC) [2], Cross-Orthogonality Check [3] and Frequency Response Assurance Criterion (FRAC) [4]. Some of these algorithms require model reductions which may produce further inaccuracies [5]. A significant amount of literature is available on these techniques, including proposals for new parameters [6], and of particular note is the work of Avitabile [7, 8]. There are specific standards commonly employed in the space industry and related thresholds for the various correlations parameters $[9,10]$ and if the level of correlation is deemed inadequate, a FEM update procedure is performed, where the FEM is amended to improve its capability to match the experimental results and reach the required thresholds to consider the FEM as validated $[11,12]$.

From a conceptual point of view there should be a first set of "identification" tests to retrieve the physical hardware dynamics (e.g. relevant modal parameters such as: resonance frequencies, mode shapes, damping etc.) and ensure a match with FEM prediction - possibly followed by a FEM model update to improve the quality of the match. Once it is established that the FEM gives an appropriate representation of the physical hardware behaviour there should be another set of "simulation" tests to reproduce the launch environment with some margins, and ensure that the hardware can withstand such environment without specific deteriorations of its mechanical performance.

In practice during single test campaigns (satellite base driven shaker/slip table test) it is aimed to achieve both goals (identification and simulations) with some initial test runs conducted with low input level to verify the behaviour of the hardware (sine surveys), followed by intermediate and full level runs to reproduce the load levels characteristics of the specification/launch. Most often these full level runs will be modified (with notches) to avoid significant exceedances of the load levels calculated during the CLA. However there are various potential complications, briefly outlined below, which hinder a blind application of these relatively simple test procedures.

Firstly, in the context of flight loads prediction and CLAs, a key point to introduce is the validity domain of the mathematical model with respect to the particular prime concerned. To clarify, of primary concern to the launcher authority is the need for representative coupling between the satellite and launcher vehicle. In this instance the need exists to obtain mathematically representative forcing functions or input boundary motion inputs into the satellite. Such conditions are generally obtained through accurate satellite modal effective mass description so that the coupled dynamic mass with the launcher is accurate. Local modes within the satellite of low effective mass would not be expected to perturb boundary input between the satellite and launcher but such modes are of clear interest to the satellite prime authority who must demonstrate that such local responses of equipment are acceptable for flight. In 
other words, the domain of correlation and particularly the focus on modes down selection for correlation is somewhat different depending upon the authority.

Secondly, in terms of issues concerning the FEM correlation, these range from the fact that achieving the correlation thresholds specified in the standards (e.g. MAC> 0.95 for the fundamental mode of vibration) is not necessarily a guarantee of a "good" model (at times a model which does not meet some of the standard thresholds might be better at predicting some responses than a model which meets the standard requirements [13]) to the fact that during testing the "boundary conditions" (from the stiffness of the shaker interface to the parameters of the controller) can significantly affect the behaviour of the spacecraft during tests and, because these parameters are not generally included in the FEM simulation, the results of the correlation may become questionable.

In addition there is the issue that, because the typical base-driven one-axis-at-the-time vibration testing does not properly reproduce the real tri-dimensional 6 DOFs launch environment, this mismatch forces the inclusion of margins, most notably because the sine test is purely dynamic and the flight loads are superimposed static plus dynamic resulting from steady state longitudinal thrust. To a certain extent, the inclusion of margins to compensate test / launch environment mismatch produces an over-designed spacecraft, sized to pass the test on the shaker rather than the real launch. To achieve a specified level for one parameter (say interface bending moment), might unavoidably produce levels much higher than expected during flight for another parameter (e.g. acceleration of a subsystem). The possibility to carry out simultaneous multi axial base driven vibration tests has been considered for several years using for example the HYDRA test facility at ESTEC [14]. Here the difficulties involved with the multiaxial control system and the inevitable dynamic coupling which occurs between large satellites and test facility have hindered a routine use of this facility for satellite 6-DOFs vibration testing. More recently a similar facility has been developed at NASA Glenn (NASA's Space Power Facility [15]), and used for the NASA's Orion Spacecraft. Various smaller systems exist $^{5}$, also available commercially, however these multi degrees of freedom tests are still the exception and typically all satellites and subsystems undergo one-axis-at-the-time type of testing, which is the reason why in this work we focus the attention on this type of tests, although a section of this article deals with the case of the 6-DOFs simultaneously testing.

The need for an accurate numerical-experimental correlation process to reduce uncertainties and margins due to test equipment deviations from perfect ideal behaviours can be addressed implementing Virtual Shaker Testing (VST)

\footnotetext{
${ }^{5}$ https://www.imv.co.jp/e/products/vibrationtest/axis/6dof/
} 
and although its principles and use have been already reported [16-19], in this paper, we give further specific background on its motivation and system level view of its implementation as a pre and post vibration testing tool. In addition the article proposes a new process and associates data flow between satellite manufacturers, test houses and launch authorities, which still respects the Intellectual Properties of the various entities, but at the same time produces a technical solution to handle the CLA related processes which is technically superior to the current practice.

In the past, a few implementations of virtual testing procedures are presented in the literature. Liu, Xiang et al. $[20,21]$ divided the facility into five different subsystems (control system, power amplifier, shaker, acceleration transducer and filter/amplifier) proving how close results are comparing the computational outcomes with the real test data. Ricci, Peeters et al. [22] showed the need for a VST extension of the computational activity using the ESA QUAD shaker [23] as test case, a further prelude of the content of in this article.

In this paper, the following Section II describes VST; including subsection A to describe its motivations and modelling of the facilities, subsection B to discuss VST usefulness in the pre physical test activity, subsection C discussing VST as post physical test tool, and is concluded with subsection D discussing its use in the FEM update process [13]. Section III addresses the case of VST for 6-DOFs testing and in Section IV, a parallel is drawn between VST and CLA proposing a new philosophy for the design and verification process. The paper is concluded with section $\mathrm{V}$ that briefly summarises the main points of the article.

\section{Virtual Shaker Testing}

We can define Virtual Shaker Testing (or Virtual Vibration Testing,) the complete end-to-end software simulation of the vibration testing process of an item (satellite or subassembly), which includes the dynamics of the test facility equipment (mechanical and electronic components, i.e. shaker, slip table, controller etc.), using the parameters (equipment settings, inputs, outputs etc.) as in a real physical test, to obtain a more accurate computational reproduction of the physical test.

The mathematical analysis underpinning the software simulation is carried out in the time domain and even on semi standard PC (CAE work station) the simulation can typically run close to real time, giving the users the possibility to monitor the same parameters that would be monitored during the physical tests, e.g. signals from the accelerometers, or Force Measurement Devices, voltages and current drawn by the shaker, and so on. As the graphical user interface 
can be identical to that of a standard shaker controller software the user running the tests would have the same "experience" as if running a real physical test, hence the name "virtual" testing, as virtual reality simulation/reproduction of a physical test.

\section{A. Motivation}

Although most test houses across the world have excellent, state of the art, equipment, the physical reality of the materials and mechanisms/equipment used, with finite values of mechanical stiffness, and electronic hardware and software which also has some limitations, from non-linearity of components [24] to delays in the software, contribute to produce differences between a physical test and the structural analyses (e.g. evaluation of frequency response functions). One of the reasons for the mismatch is that the analysis typically implements "ideal" conditions, like infinitely stiff boundaries or steady state responses, hypotheses which are not always completely verified during the physical test.

The reason for such mismatch between system test and reality of flight is primarily due to historical limitations, namely the 'test machine' (electro-dynamic linear shakers) and the other technology limits of the past (control or predictive software) which has somewhat dictated current practise.

For example Figure 1, Figure 2, and Figure 3 show typical examples of deviation from the ideal conditions due to the unavoidable flexibility of the boundary. In the first case the lateral pilots signals ideally should be zero, as the base of the craft should only move along the driven direction $\mathrm{X}$, whereas it is possible to see a significant vertical acceleration (along Z) peaking at about $47 \mathrm{~Hz}$ and $68 \mathrm{~Hz}$ which means that there was a rocking at the base of the craft that could be due to the out of plane compliance of the slip table (or the Force Measurement Device, depending on the exact position of the accelerometer). Similarly in Figure 3, which shows a longitudinal test, it is possible to notice that whilst ideally the 4 vertical pilots should have identical signals as the base of the spacecraft should only translate vertically, in reality when the spacecraft resonates, there is a rocking motion of the base, shown by the difference between pilot signals occurring at approximately $45 \mathrm{~Hz}$. 


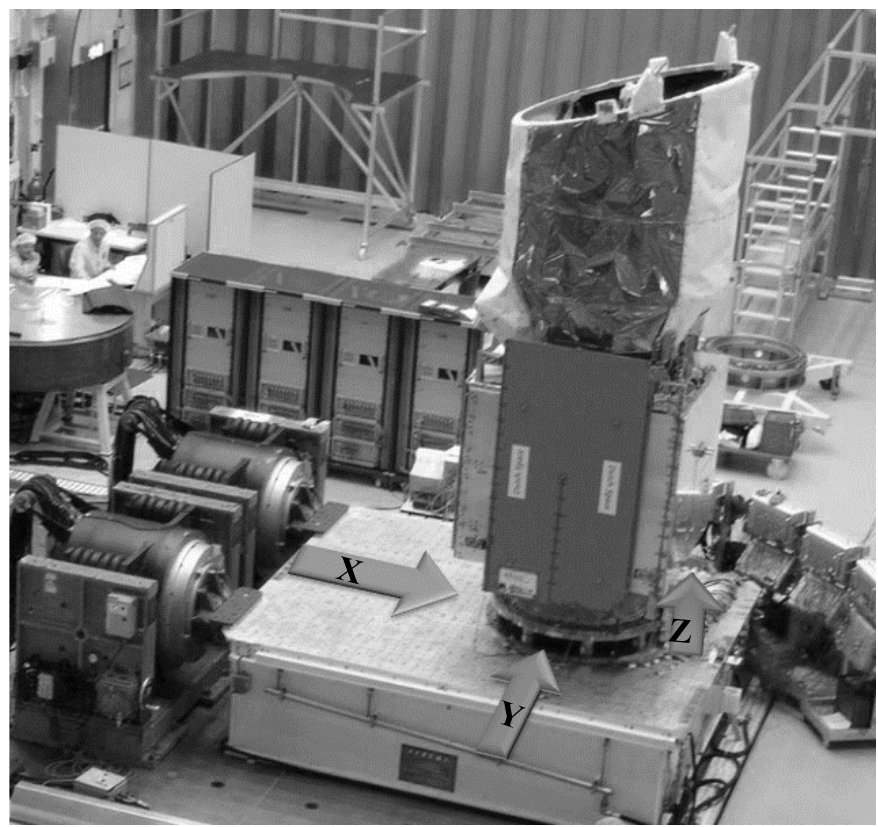

Figure 1: Typical configuration of satellite mounted on a slip table for lateral (X-axis) test

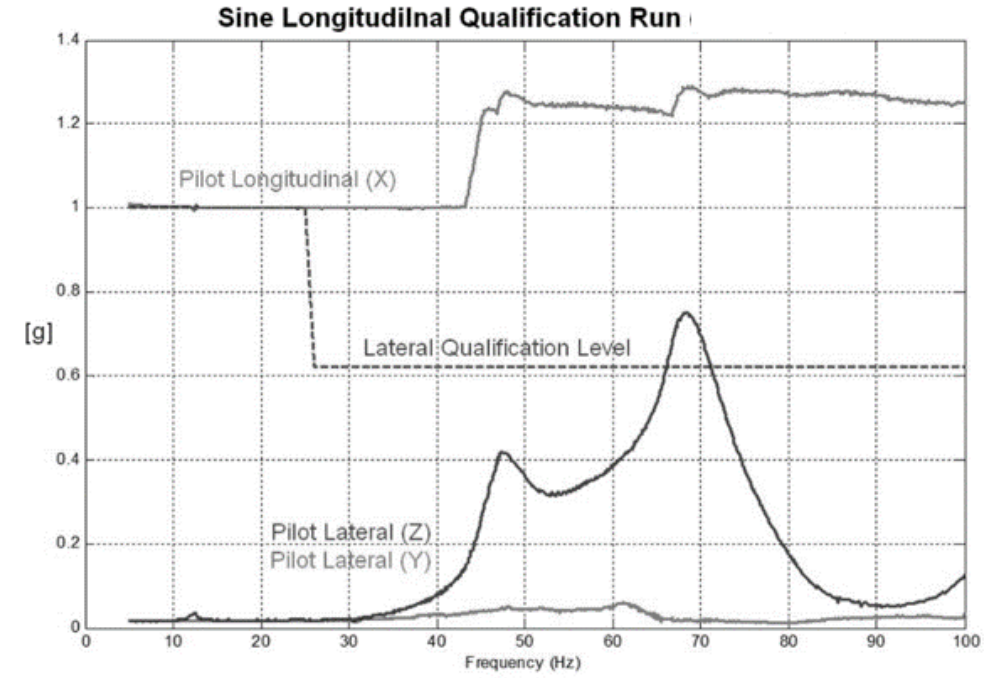

Figure 2: Example of signals from pilots, in axis (in the plot called longitudinal $\mathrm{X}$ ) and cross-axis (lateral) for a lateral axis test. (Courtesy of Thales Alenia Space) 

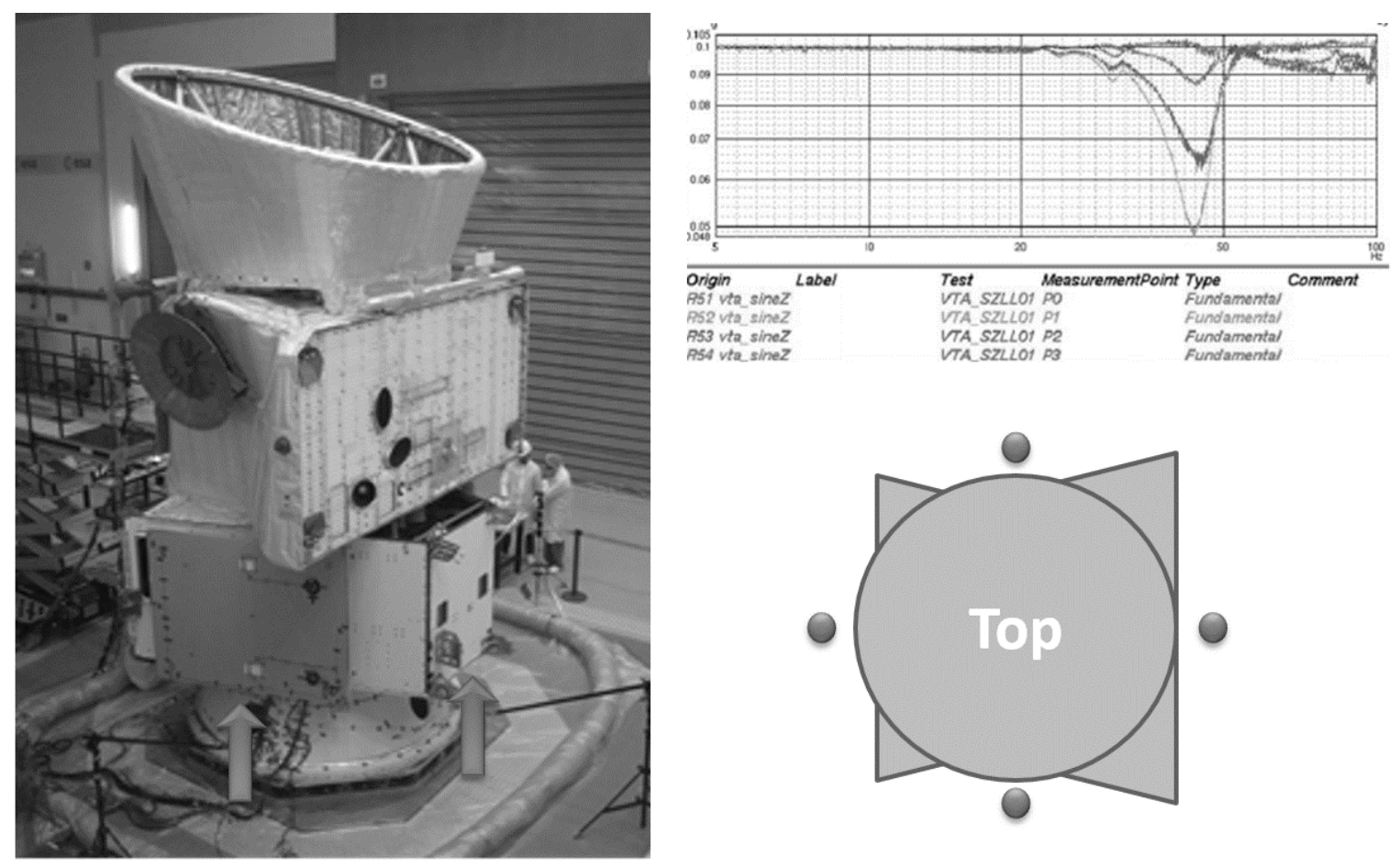

Figure 3: Left: Typical satellite during longitudinal testing. Top Right: examples of signals from 4 base pilots (accelerometer positions illustrated by the four points on sketch bottom right)

The relative level of on-axis input and cross-talk is not solely a function of satellite mass or the facility considered. Figure 4 summarises pilot injection versus cross-talk (lateral response on the pilot plane) for three different spacecraft of significantly differing masses at two different facilities with notable different longitudinal shaker set-ups. In the three cases shown for such longitudinal tests there are spectral domains where the lateral cross approaches or exceeds the desired input longitudinally.

With reference to the examples illustrated by Figure 4 a key point of note is pilot plane offset with respect to the test article's rotation apparent pivot point. Typically for spacecraft tests, piloting is specified on the Launch Vehicle Adapter (LVA) interface plane which is the reference plane considered between the launcher and satellite. In the case of 'Satellite 'A' (BepiColombo) in Figure 3, the offset is significantly exaggerated by the height of VTA. In this case it was estimated that the centre of rotation was some $1.7 \mathrm{~m}$ below the LVA pilot plane. 


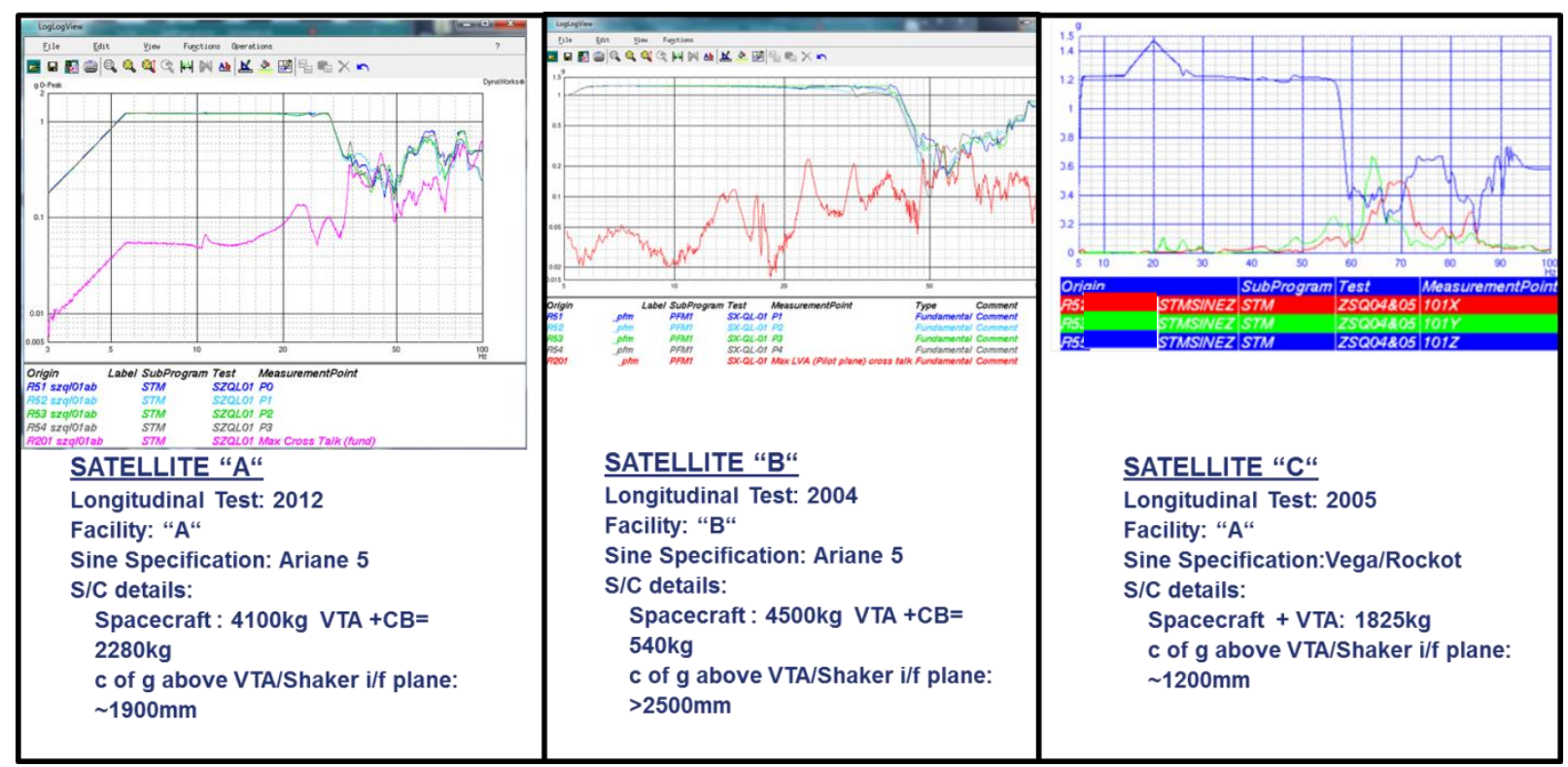

Figure 4: Pilot signals versus cross-talk for three different spacecraft of differing mass at two different facilities (during longitudinal test)

Recognition of this is important since it is possible that the facility and satellite prime could eventually be at crosspurposes with consideration of specification constraints for the test. The satellite prime will often specify desire for cross-talk on the pilot plane to be minimal and as shown here this is far from the case and exaggerated once high VTA offsets above the shaker mounting plane are introduced. On the facility side, interpretation of the satellite prime's need for cross talk levels is often viewed as the level of cross-talk on the shaker interface plane which does not account for VTA offset. What is clear from this discussion is that the physics of shakers longitudinally will almost always preclude the possibility of minimal low percentage cross-talk for the entire frequency domain and lastly both parties must be clear of the planar offset when discussing cross-talk.

The previous discussion related specifically to the longitudinal test, Figure 5 shows the same tests but for the typical lateral cases in terms of comparing on-axis pilot injection versus maximum cross-talk. It can be seen the situation is somewhat improved but in narrow band the slip table with the VTA is not infinitely rigid out of plane. 


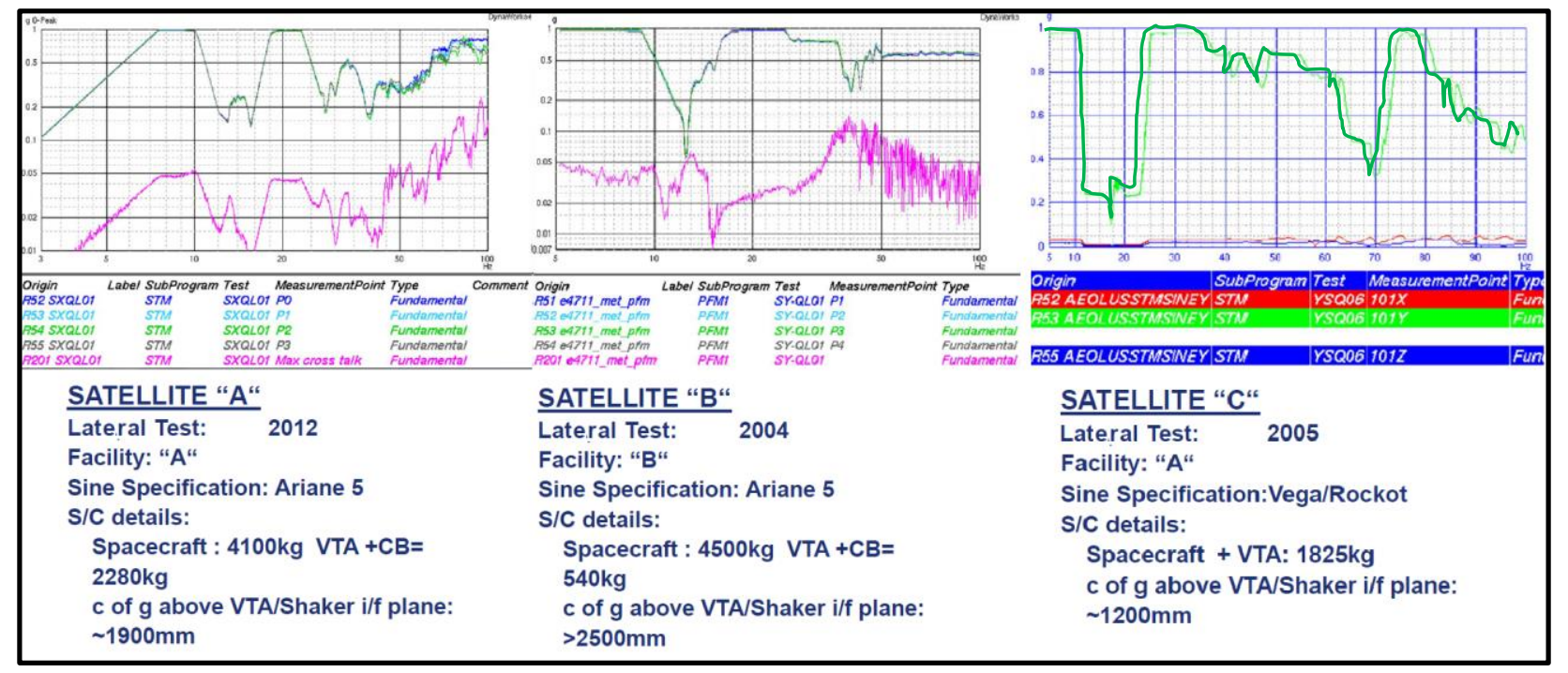

Figure 5: Pilot signals versus cross-talk for three different spacecraft of differing mass at two different facilities (during a lateral axis test). The same item configuration cases as Figure 4

Other issues relate to non-linearities seen from some of the signals from the accelerometers (see Figure 6), where it is possible to see different harmonics contributing to the response. In such case illustrated here, which refers to a cross axis signal of an accelerometer closed to the base of the craft, it is clear that the desire for monochromatic or pure single harmonic motion is clearly deviated from.

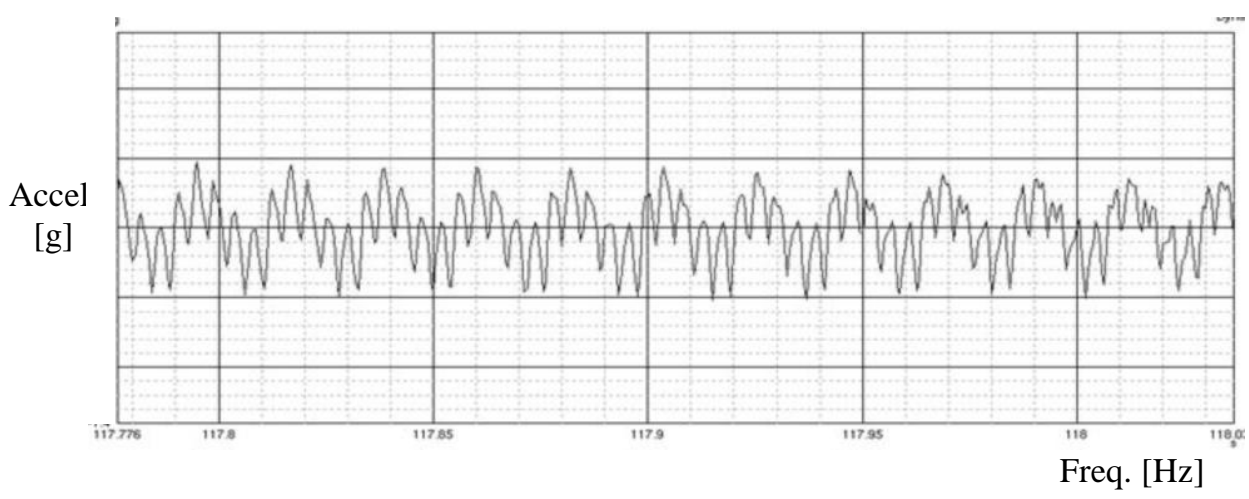

Figure 6: Time domain accelerometer signal (cross axis) during sine tests where it is possible to see other harmonics superimposed to the ideal sinusoidal signal

Indeed sometimes a mismatch is just the result of the analysis approximation, e.g. in a real sine sweep test, depending on the speed of the frequency sweep, damping and other parameters, at resonance we might not develop the full amplification expected from a steady state response calculation (like it is implicit in FRF calculations). Or after resonance we can observe a beating phenomenon that theoretically is well understood and documented but in 
practice these effects are most often ignored during the standard analyses and following satellites numericalexperimental correlation exercise. All of these points support the need for VST where it was previously stated that such a tool can help the system teams understand the causes of such observations and the relative influences of each specific parameter or mechanical architectural elements in the test set-up which promote observed test findings.

From a practical perspective, going to test ignoring issues like those mentioned above can produce significant mismatches with the expectations deriving from the typical structural analysis carried out during the spacecraft development, resulting in expensive delays during tests and even aborts commanded by the operator or automatically triggered by the controller. Hence a pre-test activity as that illustrated in the section below can significantly de-risk the physical tests and ultimately save time and money.

Considering then post-test FEM correlation, it is fairly obvious that, because the physical test results may well be affected by the kind of issues mentioned above (e.g. flexibility of the boundary that ultimately produces a resonance frequency lower than that obtained for a rigid/clamped boundary), to ignore these effects in the $\mathrm{FE}$ analysis and satellite FEM correlation/update would ultimately produce an incorrect Satellite FEM. This issue can be resolved using VST during the FEM correlation process, as illustrated in the section below.

\section{Model of the facilities}

VST relies on the availability of a very accurate mathematical model of the test facility, hardware and software. In this article we have focussed on the facilities available at the ESA test centre, but this should not be viewed as a limitation since the inherent interfaces within the VST tool can be broken and replaced by modules (e.g. shaker dynamics) specific to the other major spacecraft test facilities. The mathematical models modules have been assembled from FEM of the test facility partially built in house and partially sourced by sub-contractors along with a control module representative of the facility controller. The FEM of the mechanical part of the test facilities are shown in Figure 7 and Figure 8. The FEM have to be reduced in state space and are then coupled with the rest of the model of the facility. The model of the electrical/electronic part of the test facility has been directly built with Simulink, assembling a controller that has been supplied by the controller manufacturer as a black box (see Figure 9). A FE model of the shaker (seismic mass, actuators and table with relative connections) was built and validated by ESA. A modal analysis of the structure shows rocking modes of the table at about $59 \mathrm{~Hz}$, well below the ideal threshold at 100 Hz. The same happens with HYDRA facility, which has six pseudo-rigid body modes between 20 and $40 \mathrm{~Hz}$ when the table is running empty. 

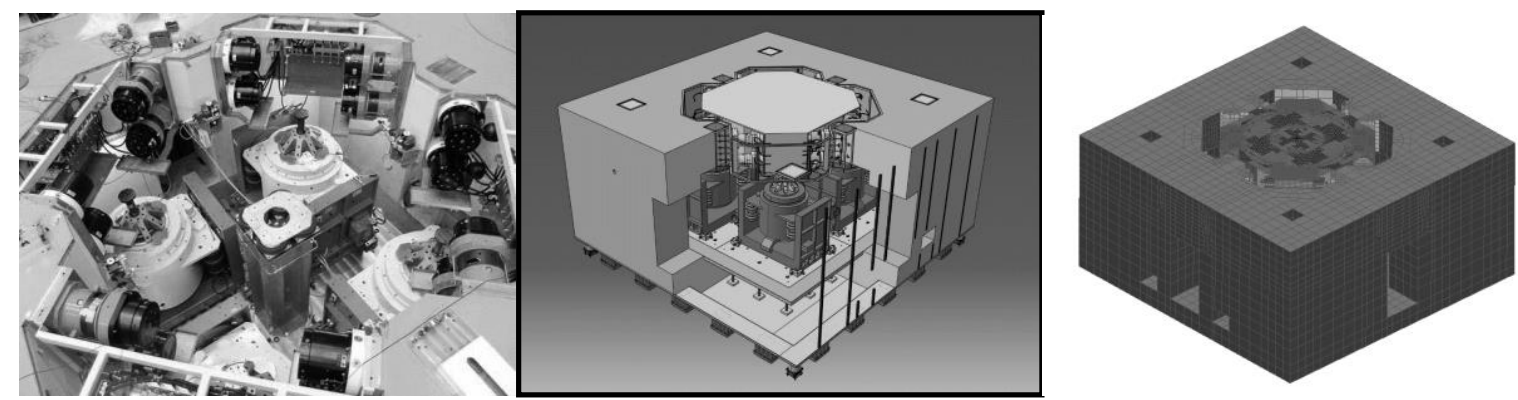

Figure 7: QUAD test facility Left picture shows the shakers underneath the table, centre picture is the CAD model of the facility, and right picture its FEM
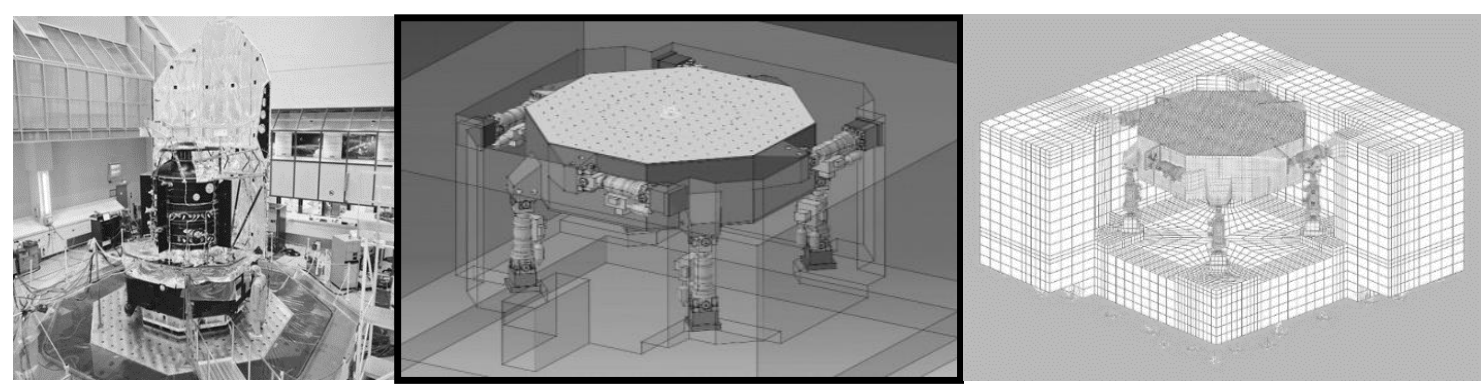

Figure 8: HYDRA test facility. Left picture shows the facility during the test of Hershel, the centre figure is the cad model showing the hydraulic shakers driving the table, and on the right is the FEM of the facility

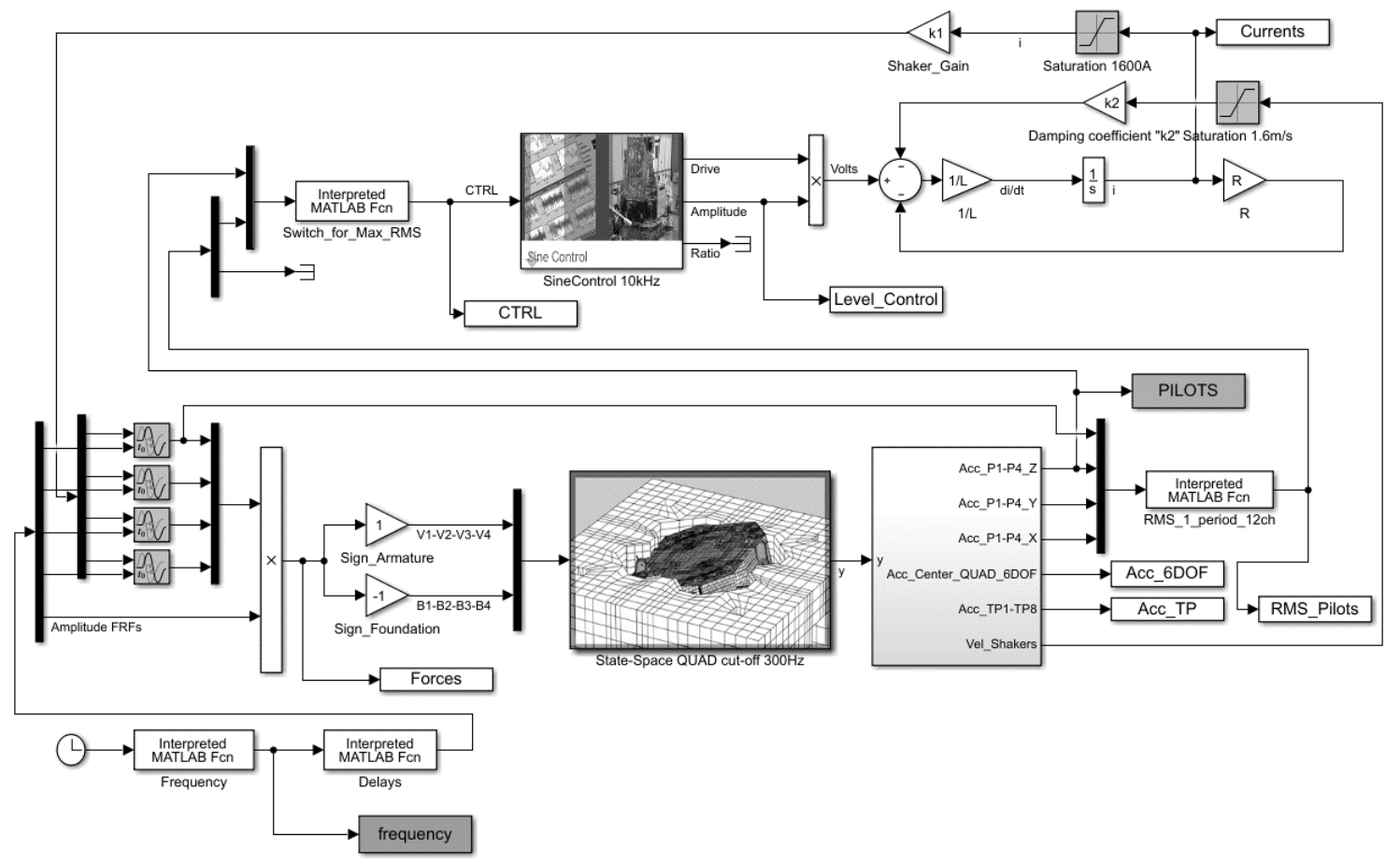

Figure 9: Simulink model of the test facility including shaker/slip table at the centre and the various electrical/electronic equipment and controller 


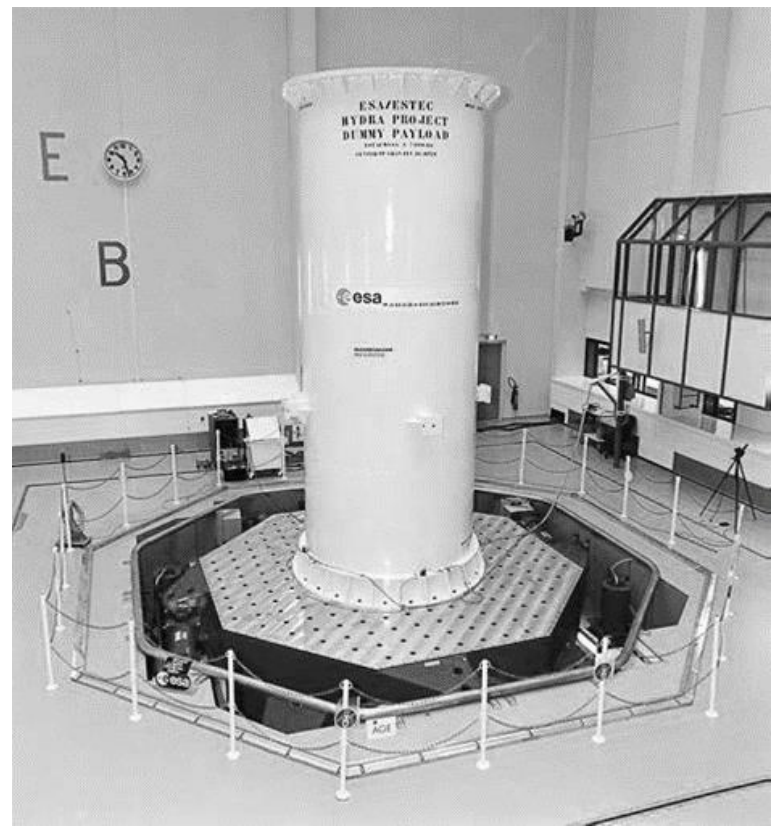

Figure 10: HYDRA mass dummy mounted on the shaker for the validation of the model of the test facility
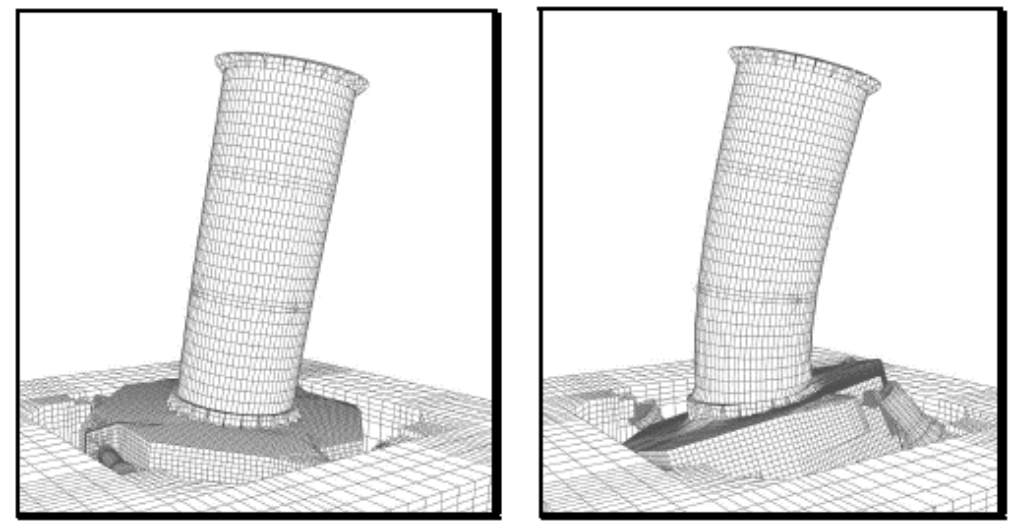

(a)
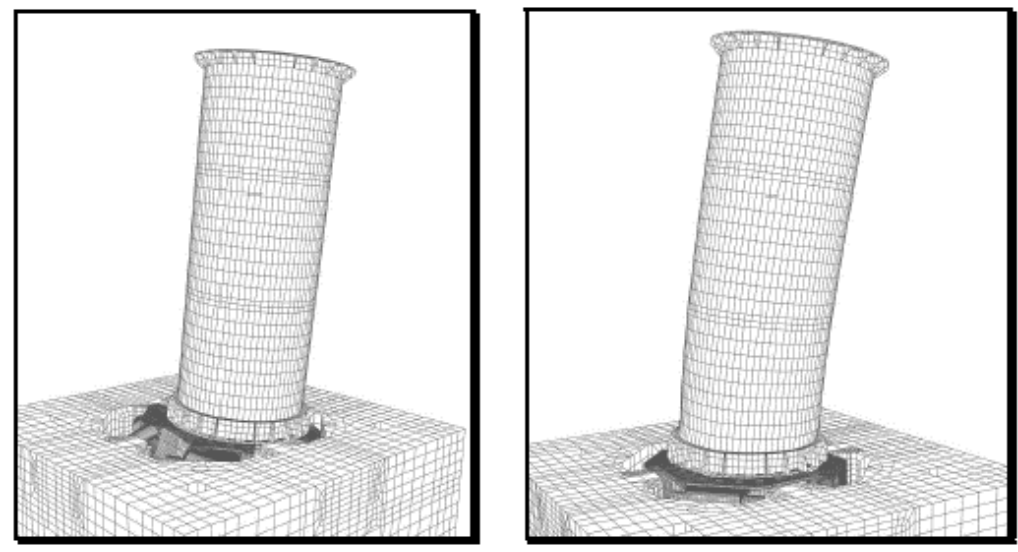

(b)

Figure 11: Mass dummy dynamic behaviour on HYDRA (a) and QUAD (b) shakers [25] 
A correlation of the mathematical model of the test facilities has been carried out performing physical tests using a mass dummy with known dynamical behaviour on the shaker (see Figure 10), and this has allowed to verify the quality of the FEM of the shaker and control system, which are the essential prerequisites for VST. Figure 11 shows the behaviour of the mass dummy on both shakers. In both cases the main bending mode of the structure splits into two different modes, but at different frequencies for each test case, as the two shakers have a completely different design, functioning mechanism and dimensions. If on one side it seems that this makes the problem more complex, in reality this is a further proof that any facility can be modelled and utilised as virtual testing tools. For more information on HYDRA and QUAD shakers' models and validations see [25-27]. Also other authors have modelled similar systems as shown in [28-30].

\section{B. VST as pre-test tool}

VST can become a fundamental tool to be used during the pre-test activities as the capability to produce accurate predictions of the dynamics which are produced during the physical test will significant de-risk it. On one hand the design of the test input profile and its notches will be more reliable and less subject to last minute changes, during the physical test run, when mismatches between ideal FEA and real test signals are typically detected. For example, the controller cannot follow the ideal/envisaged test profile and the slope or depth of a notch has to be modified on the fly without appropriate time to examine all its implications. The capability to better predict cross responses (e.g. due to shaker or slip table compliance) also help to prevent other signals reaching unexpectedly critical levels, triggering further automatic notches or jeopardizing the spacecraft integrity. In this section an example of how the predictions can become significantly more valuable when introducing virtual testing is shown.

As the test is longitudinal, cross-axes effects are expected to be relatively low and this can also normally be observed when running finite element analyses on the spacecraft model. In reality, the measurements taken by the pilots placed on the four corners of the shaker table show non-negligible later displacements. In order to understand the magnitude of cross-axes effects, a virtual testing run can be sufficient, without putting the satellite under any real stressing situation.

In this case we have considered the FEM of BepiColombo (one of Airbus' large scientific spacecraft, built for the European Space Agency's first mission to Mercury) mounted on the QUAD shaker at the ESA test centre (see Figure 12 for the real test and Figure 13 for the FEM). This is reduced into state space matrices in order to run the Simulink model representing the real facility (see Figure 9). 


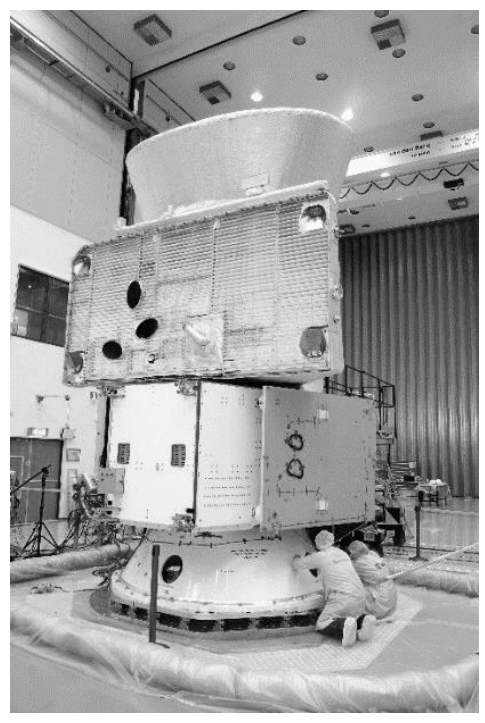

Figure 12: BepiColombo tested on QUAD Shaker at the ESA Test Centre

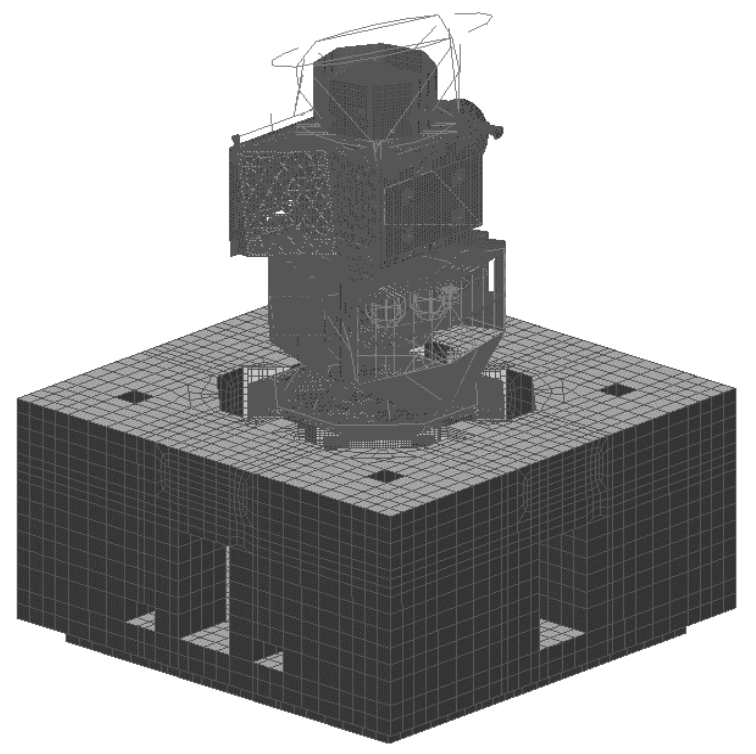

Figure 13: Coupled FEM of BepiColombo and QUAD Shaker

Figure 14 shows the prediction of one of the pilots in the lateral direction when a vertical acceleration is applied at the base. The blue curve is the "virtual testing" prediction, the yellow curve is from the real test data and the red curve is the prediction using only BepiColombo model (the pilots were placed at the top of an interface between the table and the spacecraft). As it can be observed, the real lateral measurements are between 2 and 3 orders of magnitude higher than finite element predictions and this is relatively well matched by the virtual testing data. 


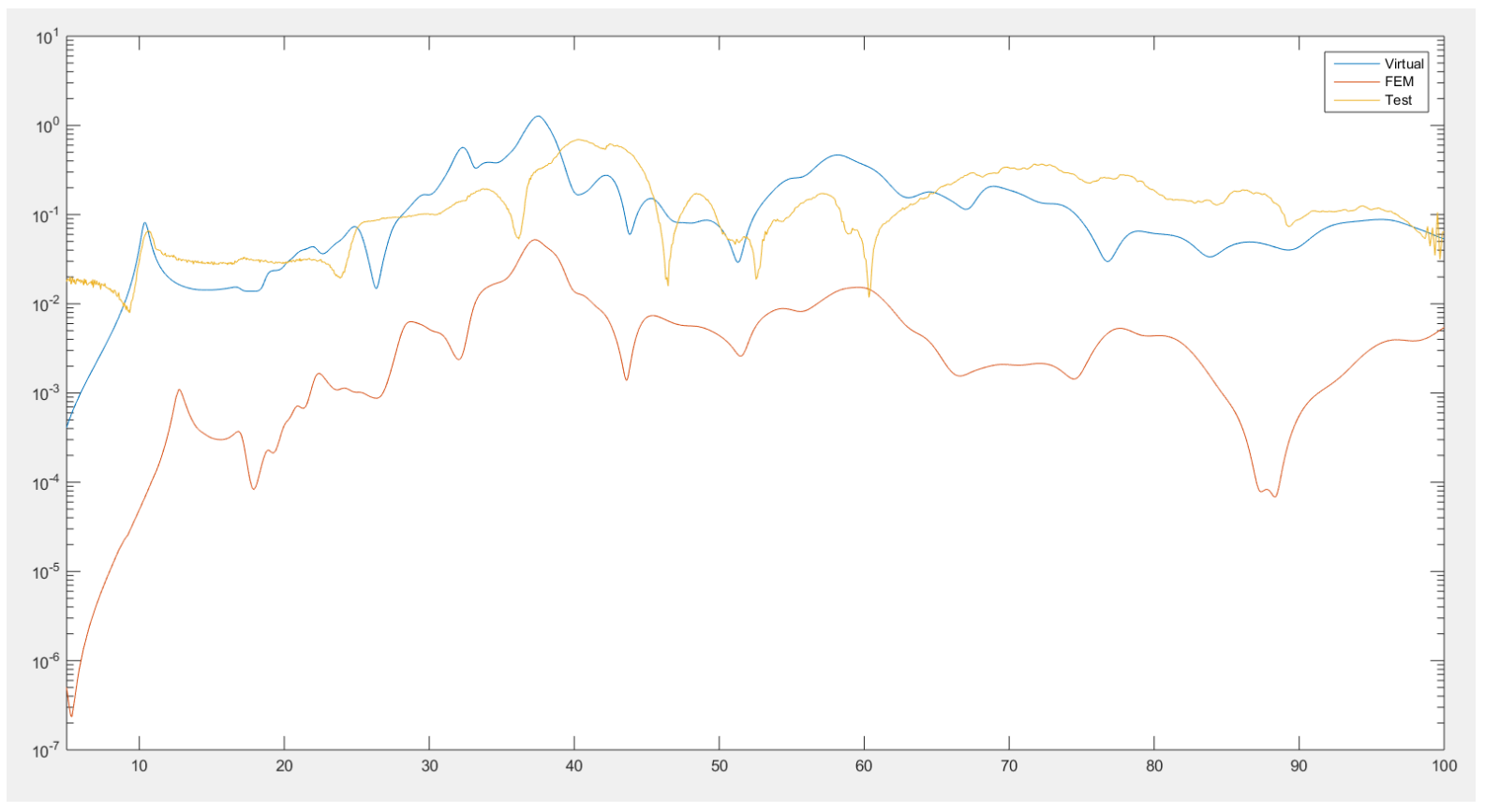

Figure 14: Lateral response of one of the pilots (from VST and real measurements + FEM predictions)

These lateral displacements might trigger unwanted behaviours which normally would be detected only during the physical tests, with potentially disastrous consequences. VST runs well in advance of the physical test campaign would give time to plan and take appropriate remedial actions.

The capability to accurately calculate the coupling between specimen and test facility to predict the real behaviour of the satellite on the shaker at times can also have the advantage to allow the use of a smaller facility where strong coupling would be expected, which would normally suggest that the facility is inappropriate. If VST shows that all the values of the relevant parameters during the test are acceptable for the purposes of the test there is no reason why the smaller facility cannot be used rather than a larger more expensive facility. This real situation has actually occurred in Industry and has been reported during the 2017 Virtual Shaker Testing workshop held at the European Space Agency [31].

\section{VST as a post-test tool for model correlation}

VST allows to reproduce in the analysis/simulations most aspects of the physical reality of the tests (e.g. stiffness of the shaker slip table etc..) so these effects can be filtered out from the correlation process of the satellite FEM and because the shaker/slip table, controller models etc. (whose models are included in the VST procedure) are already 
correlated and validated with high level of accuracy, the potential mismatches between test and computations will only be due to inaccuracies of the Satellite FEM.

A modal analysis of the coupled spacecraft-shaker system already shows important changes in terms of dynamic behaviour of BepiColombo, starting with the fundamental bending modes, that dropped approximately $20 \%$, from 12.7 $\mathrm{H}$ and $13 \mathrm{~Hz}$ form standard modal analyses performed with BepiColombo alone, constrained to the ground, to 10.4 and 10.6 Hz obtained from BepiColombo mounted on the shaker. Similar effects can be seen on the other mode shapes, with a total of $39 \%$ of modes affected by the coupling with the shaker up to $65 \mathrm{~Hz}$ (see Table 1). As can be seen by Table 1, just analysing the satellite mounted on the satellite mounted on the shaker has increased the number of modes calculated in a range up to $65 \mathrm{~Hz}$. Although some modes of the satellite were not affected by mounting this on the shaker, some $26 \%$ of the modes showed a significant change in their frequency, and about $13 \%$ of the satellite mode shapes are significantly different between the two configurations.

\section{Table 1: Natural Frequencies of "Only Bepi" and "Bepi \& QUAD" models}

\begin{tabular}{|c|c|}
\hline Resonances Bepi base clamped & 169 \\
\hline Resonances Bepi mounted on QUAD & 189 \\
\hline Significant Change in Frequency & $50-26 \%$ \\
\hline New/Different Shapes & $25-13 \%$ \\
\hline Total & $39 \%$ \\
\hline
\end{tabular}

Running the full virtual testing simulation further increases the differences from a nominal FE analysis on the spacecraft as also the controller effects are now included. In order to quantify the actual changes, two correlation criteria are here used: RVAC and FRAC [32]. RVAC is very similar to the better known MAC, but instead of comparing mode shapes, Operational Deflected Shapes (ODSs) are compared. The reason for using ODSs is that the response at a specific frequency for each relevant DoF can be easily computed performing an FFT of the time domain signals produced by the VST simulation. Alternatively, it is possible to "follow" the time domain signals of the relevant DoFs. with a tracking filter synchronised with the input, exactly as it would be done during a physical sine sweep test, and filtered and unfiltered "FRFs" can be produced, and used to generate deflected shapes at the relevant frequencies. Because this would be exactly the same data processing algorithm that is used to generate the FRFs from the physical tests (where the raw signals form the runs are clearly generated in the time domain) the comparison of the ODSs (computational, from VST, and physical tests) is not influenced by the algorithm for the data processing. 
However there are situations where to compare mode shapes is more appropriate, and in this case the results from VST have to be processed with the same algorithm that is used to reconstruct the mode shapes from the FRFs generated during the physical tests. Indeed this increases the effort involved in VST with respect to a standard FE modal analysis. Another reason for using ODS is that they are proportional to the response and ultimately to the margins (e.g on stresses and accelerations) in the design, regardless of the fact that the response at a frequency can be produced by the summative effect of various modal responses. For this reasons ODS are not useful to shed lights on the modal behaviour of the specimen, which is useful during a FEM update process.

In this section, a comparison of ODS is used to work out origins of the mismatch between test and computations, i.e. whether this is due to the coupling with the shaker, to the control system or to actual inaccuracies in the satellite FEM. FRAC compares two frequency response functions and it is here used to quantify how important the improvement is when introducing virtual testing to our analyses. Both correlation criteria produce an index varying from 0 to 1 , with 1 meaning perfect correlation.

(a)

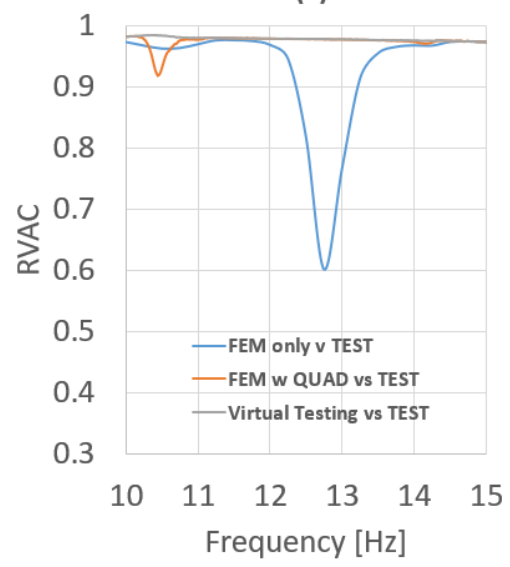

(b)

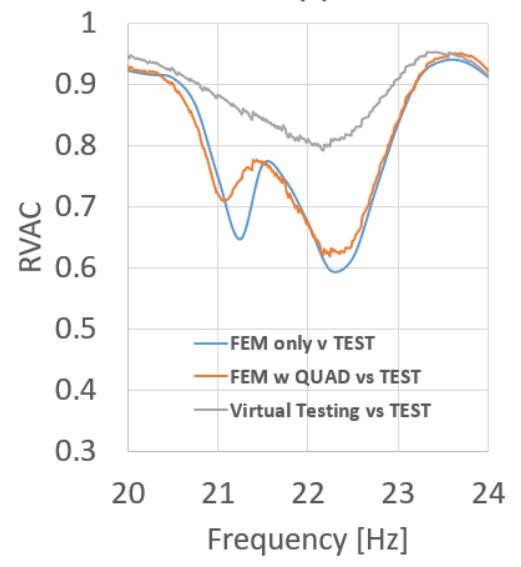

(c)

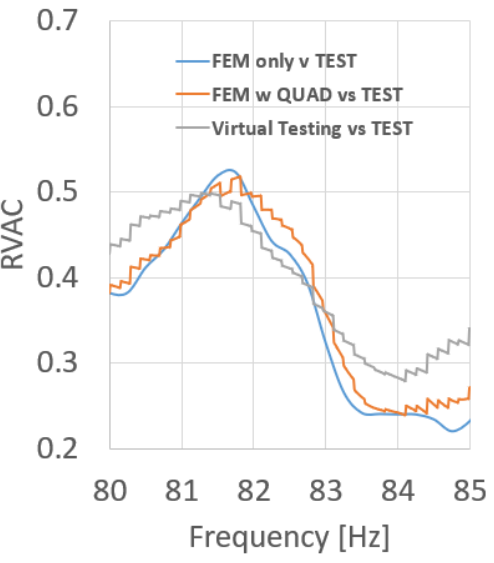

Figure 15: Comparison of RVAC values calculated for different ranges of frequencies

In Figure 15, RVAC is computed for three different cases for a range of frequencies, all of which compared to real physical test data: i) standard FRF FEA performed with only Bepi constrained to the ground; ii) analyses performed with coupled spacecraft-shaker model; iii) full VST simulation. Figure 15(a) shows RVAC values obtained for frequencies from 10 to $15 \mathrm{~Hz}$. The impact created by the decrease in frequency of the main bending modes is here clear, with the blue comparison (finite element model of only Bepi vs test) giving the worst prediction and almost perfect correlation in the other two cases. This means that the "poor" correlation shown by the standard FEA was 
because the shaker model was not included in the simulation, and including this, we achieve excellent correlation. Increasing the frequency range (Figure 15(b)), the correlation between test data and coupled finite element model also starts to show low values, whilst the full virtual testing simulation correlates well. This means that the discrepancies in the computational results when running only BepiColombo are due to control system related issues or other environmental factors, and not due to errors in modelling. Finally, Figure 15(c) depicts the RVAC values for high frequencies, showing a low correlation in all three cases, which would correspond to wrong modelling of the structure under tests, but this is to be expected when using finite element analysis as computational method for high-frequency applications.

Figure 16 shows the difference in FRAC values observed with and without the implementation of virtual testing simulations. On the $\mathrm{x}$-axis the 363 output locations considered for this study are listed and in the y-axis a positive value means that the FRAC calculated after implementing virtual testing has improved, whilst a negative value means that it has worsened. Only six output locations show a negative value, meaning that in more than $98 \%$ of the cases, virtual testing improves the correlation, and significantly as the average improvement is of about 0.2. Considering that the average FRAC was 0.6 , a 0.2 improvement corresponds to a $\%$ improvement over $30 \%$.

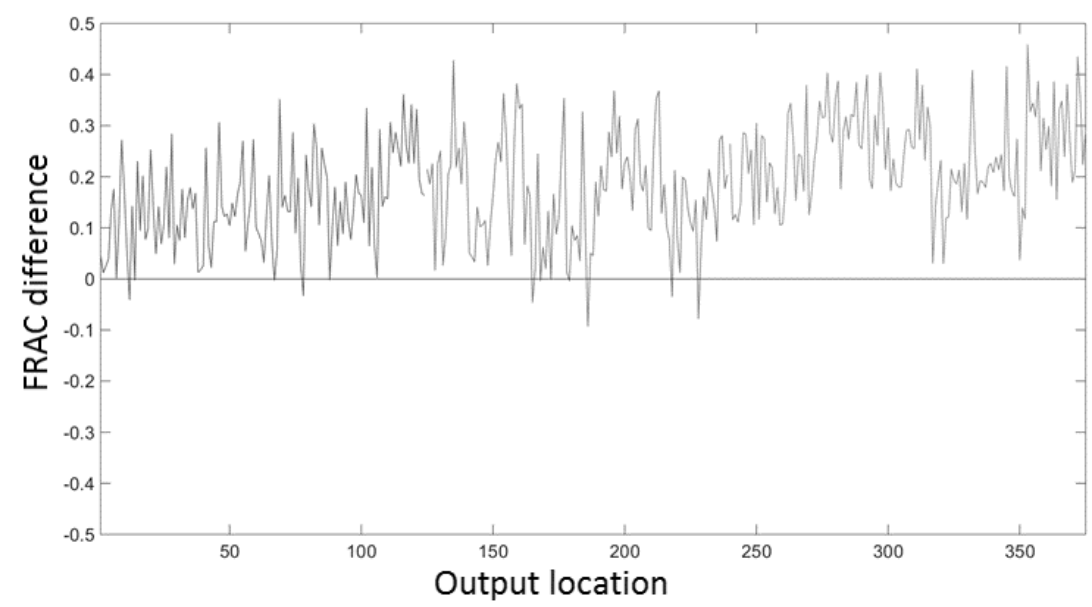

Figure 16: FRAC difference in the values when running classic analyses and implementing virtual testing

In addition, two examples of measurements of accelerometers' locations during the test are compared to the actual test results and to the nominal solution that would be obtained running a FE analysis on the sole spacecraft (Figure $17)$. 

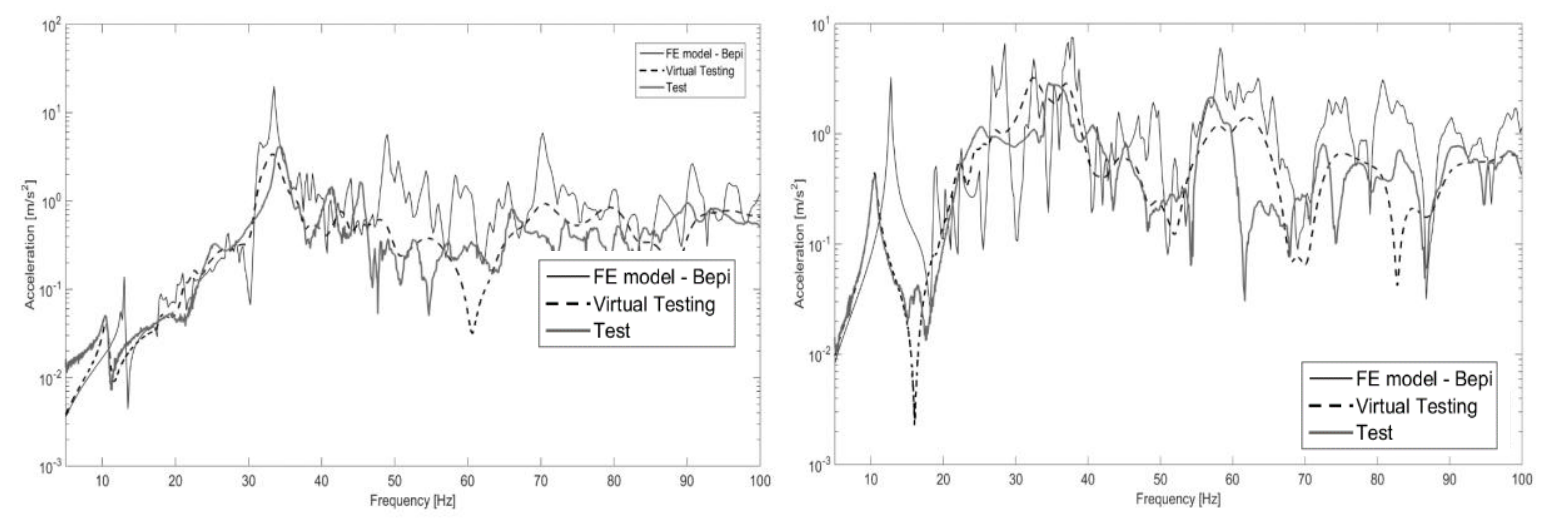

Figure 17: Examples of computational and experimental FRFs for representative output locations for BepiColombo

As the comparisons show, the first peak is now matched perfectly as expected, but also the trend across the whole frequency seems to undergo a significant improvement from the nominal solution and this is produced not only by the dynamic coupling with the shaker, but also by the control system, which is non-perfect, and other delays of the testing environment.

\section{VST as a post-test tool for model update}

FEM updating methods have been developed and tested for decades [33, 34]. More recently, a number of different strategies have been applied to the field to make the process faster and more accurate: examples are Genetic Algorithms [35], Hamiltonian Monte Carlo [36] and Component Mode Synthesis techniques [37]. Essentially these methods consist of modifying some of the parameters of the FE model, to improve the match between mode shapes predicted by the FEM and those retrieved during the physical test. Normally this exercise is performed only for a relatively small number of mode shapes (target modes) [38] that are particularly relevant. Reference to 'target modes' is important since it is not the assessment of measured quality (e.g. MAC) which is time consuming or computationally expensive, but is the process of FEM update and correction (to produce a validated FEM) which is where a significant effort might be spent, depending on the extent of the corrections to be implemented.

Typically the first few modes (with high effective mass) and the modes that are mostly contributing to critical responses (e.g. delicate payloads or solar arrays modes etc.), are taken as target modes, the basis for this was discussed previously in the context of launcher and spacecraft prime authority focus. This process breaks down if the experimental modes parameters are affected by the test equipment and its settings utilized for the runs. For example 
the FEA may consider the satellite as ideally grounded whereas in reality the compliance of the shaker/slip table will affect mode shapes and frequencies. Similarly the test could have been run sweeping at a relatively high number of octaves/min, which would not give sufficient time to fully develop the resonances thus producing overestimates of the damping. To overcome these issues the FEM update can be carried out using the whole VST model (i.e. including the effects of test facility equipment). The mode shape of the FEM in this case can be retrieved from running the VST in the time domain, and building the mode shapes from the time signals, transformed into FRF, as it is done during a normal sine sweep physical test. Here it is crucial that the raw data in the time domain produced by the VST, are treated using the same algorithms and parameters as those used during the physical tests to produce the responses in the frequency domain and mode shapes from the accelerometers time domain signals. Indeed this adds further computational complexity to the process with respect to a standard FEA, which would produce the mode shapes directly from a simple FE modal analysis. This is likely to have an impact on the schedule and cost of a project, which might be difficult to justify, in cases where the test facility is expected to have negligible coupling with the satellite dynamics (e.g relatively small satellites tested on large facilities) but for situations where significant coupling is expected (large satellites possibly on high test adaptors) with deviations from ideal conditions, the benefits should justify the shortcomings.

A very important remark is that if the effect of the facilities is not separated from the dynamics of the satellites there is the risk to update the satellite FEM artificially including in the satellite model itself the effect of the test facilities (e.g. boundary conditions compliance) in the attempt to match the test data. This would actually decrease the representativeness of the satellite FEM and ultimately jeopardise the quality of the satellite/launch vehicle CLA.

\section{VST for 6 DoFs testing}

Unexpected outcomes from a test campaign are issues which can result in some cases to damages of the tested structure with a consequent important loss in terms of money and time. It is therefore of extreme importance to be able to predict unexpected behaviour and this is exactly what VST would be able to do if used as a pre-test tool. As shown in the previous sections, in addition to the dynamic coupling between shaker and spacecraft, the non-perfect control system is also an important environmental factor which needs to be taken into account, especially when this 
becomes very complex. A perfect example is given by the HYDRA shaker (Figure 8), a hydraulic shaker property of ESA which can test along six degrees of freedom simultaneously. Using VST here before the test is highly desirable, as it is an essential proof of the feasibility of the test itself.

A virtual testing simulation with an input of 16 short transients, representative of classical CLA transient inputs, has been run in the frame of an internal ESA research activity. The payload used is a mass dummy (called TEDY in short) bolted on the shaker. The FEM of TEDY is shown in Figure 18. The control system utilized was provided by MTS, with the same functionalities both in the real test facility and in the virtual testing simulation, where it is adapted to be linked to a Simulink model.

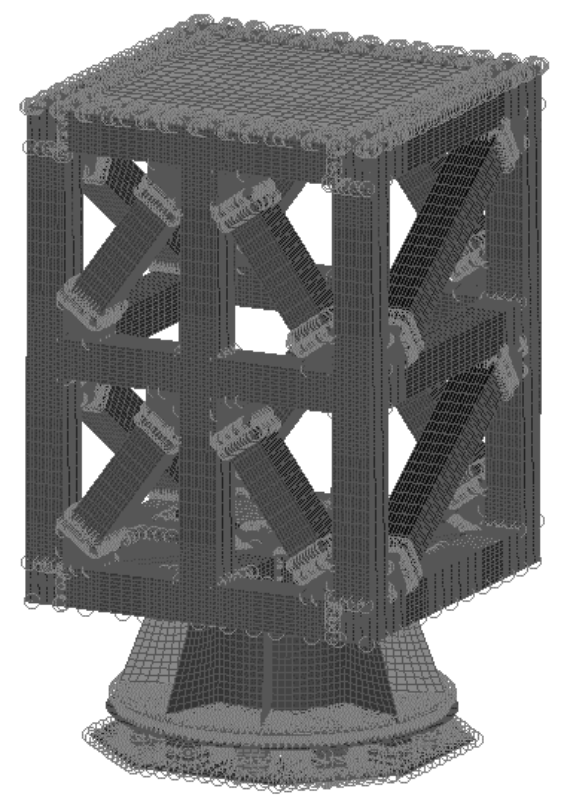

Figure 18: FEM of TEDY mass dummy

Firstly, in order to show the effect of the dynamic coupling between shaker and structure, the differences in terms of main resonant frequencies of TEDY when this is coupled to the HYDRA shaker are shown in Table 2, and they are significant despite the structure being notably smaller than the normal size of spacecraft normally tested on shakers of this size. 
Table 2: Differences in frequency for the main modes of Tedy when coupled to Hydra

\begin{tabular}{|c|c|c|}
\hline \multicolumn{1}{|c|}{ Mode } & Frequency only Tedy & Frequency Tedy+Hydra \\
\hline First bending mode & $28.83 \mathrm{~Hz}$ & $28.17 \mathrm{~Hz}$ \\
\hline Second bending mode & $30.36 \mathrm{~Hz}$ & $29.41 \mathrm{~Hz}$ \\
\hline Torsional mode & $83.55 \mathrm{~Hz}$ & $81.82 \mathrm{~Hz}$ \\
\hline Longitudinal mode & $99.43 \mathrm{~Hz}$ & $98.39 \mathrm{~Hz}$ \\
\hline
\end{tabular}
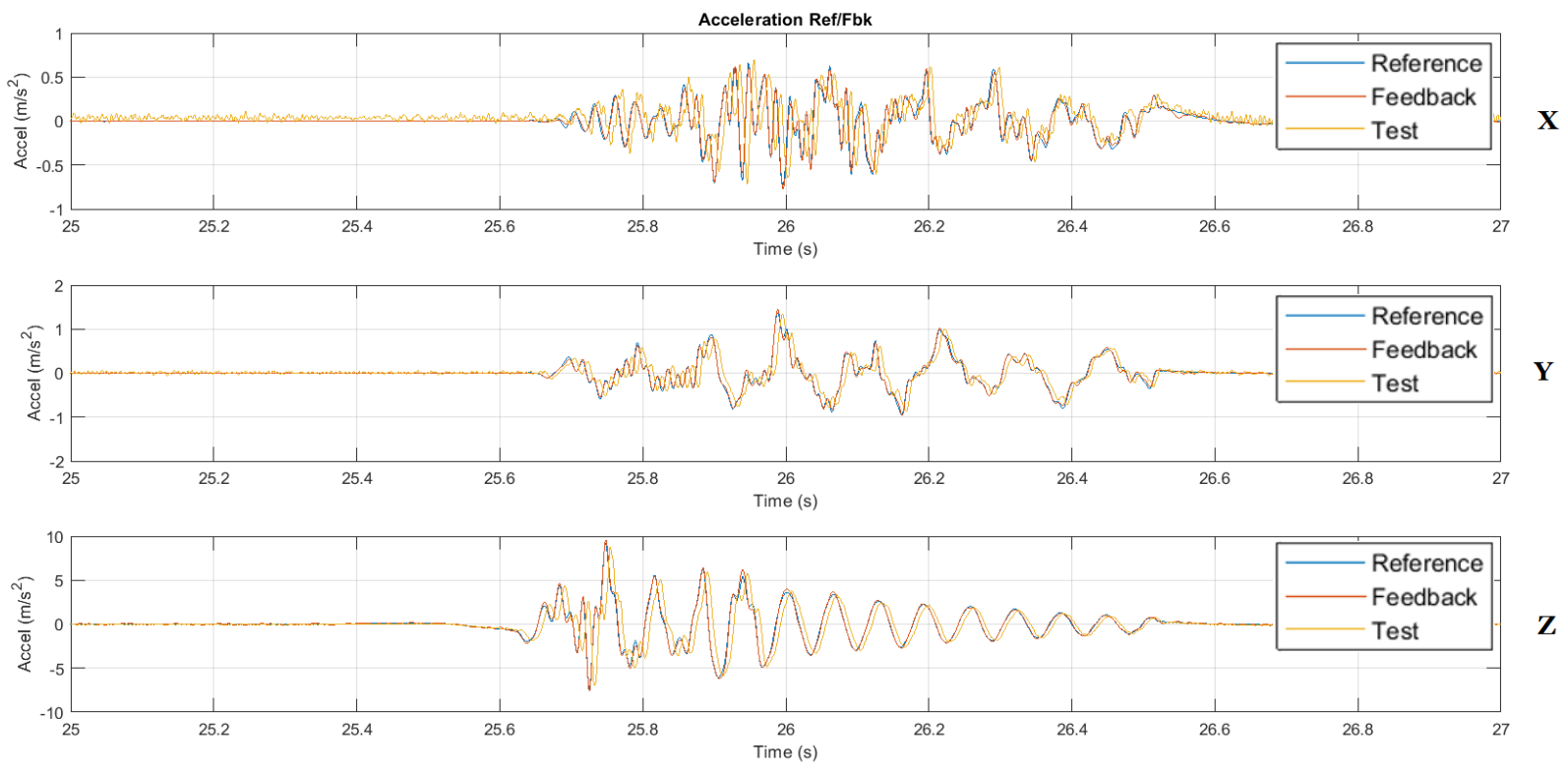

Figure 19: Reference Signal compared to Feedback Signal from VST and Test Data in the time domain

Figure 19 and Figure 20 show the results for one of the 16 short transients, both in the time and in the frequency domain. The damping used for the virtual simulation was 0.008 critical, later confirmed by the real test results. As it can be observed, a part from very small deviations, the control performs well along the three translational degrees of freedom and this is also backed up by the real test data, producing the same outcome. 

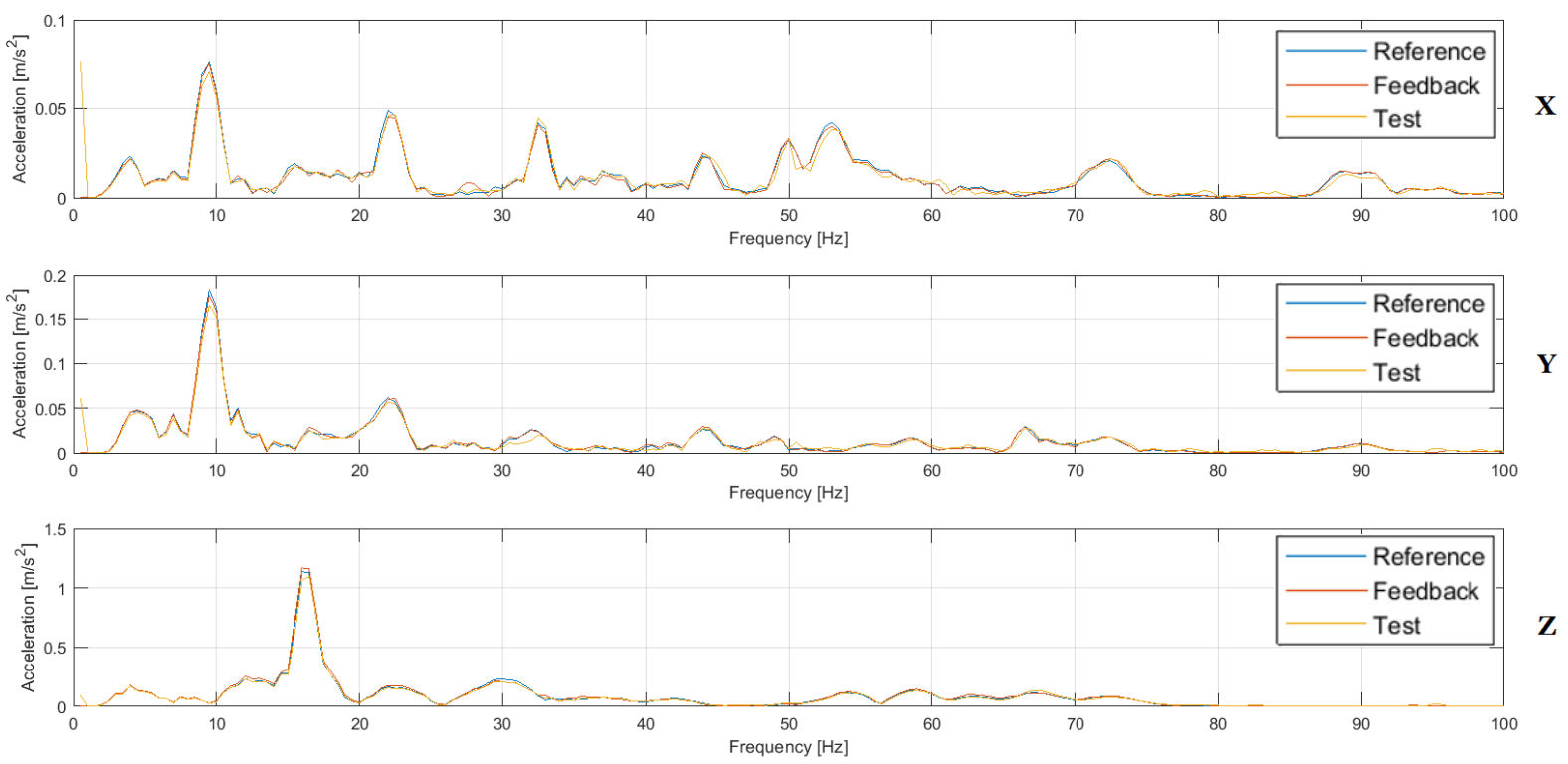

Figure 20: Reference Signal compared to Feedback Signal from VST and Test Data in the frequency domain

\section{A philosophy for implementation of Satellite/Launch Vehicle CLA and VST}

From a structural analysis point of view, the satellite launch vehicle CLA briefly discussed in the introduction is very similar to the VST procedure discussed in the previous section, as in the first case the coupled system is the satellite mounted on the launch vehicle, excited by the launch loads, whereas in the second case the coupled system is the satellite mounted on a slip table or shaker table and excited using electrodynamic or hydraulic shakers (see Figure 21).
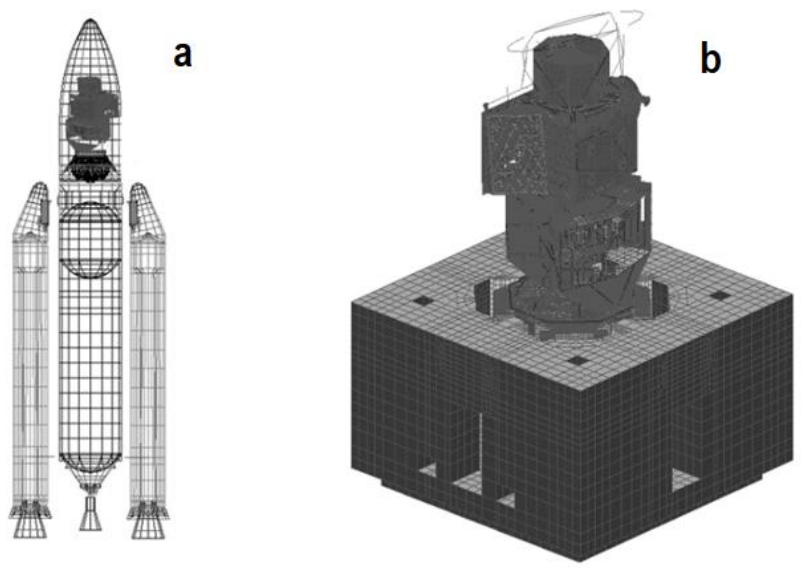

Figure 21: a) Satellite FEM assembled in the launch vehicle for the CLA, b) Satellite FEM mounted on the shaker for Virtual Testing. 
Starting with the satellite/launch vehicle CLA, the current typical scenario is the "Qualification by Interface" whereby the Satellite/Spacecraft (SC) Prime provides/delivers the satellite FEM to Launch Vehicle (LV) Authority; the LV Authority performs a CLA (preliminary) for the Flight Limit Loads (FLL) predictions and requests the Satellite prime to perform sine vibration tests (typically on the satellite Structural Qualification Model - SQM) for satellite FEM correlation and Qualification. The FEM is correlated against the physical test results by the satellite prime, possibly updating the FEM if necessary. Finally the SC Flight Model (FM) is tested (with the test procedure informed by the CLA for notches etc.) and these test results (once verified that the physical FM behaves as the SQM) are used for the final verification against the final CLA performed by LV authority.

However there are alternatives to the philosophy briefly summarised above, and one of these was the NASA "Design \& Validation" which was applied for the large payloads flying on the Space Shuttle. Here was the LV Authority that provided a reduced (valid) LV FEM and loads to payload (SC) prime, and was the prime who performed the CLA for Design Limit Loads predictions (Design CLA). The SC prime then performed SC Modal Survey Tests and Main Components (Items of Equipment) Sine Vibration Tests for SC FEM correlation/validation to FLL and Design assessment. Finally, the SC reduced/validated FEM was provided to LV Authority for the final CLA assessment (Verification Load Cycle).

The great advantage of the second philosophy (Design \& Validation) and associated procedure was that the CLA was an integral part of the Design Cycle, generating a spacecraft designed for flight. Whereas the current procedure (verification by interface) uses the CLA as a check, rather than as part of the design process, with the final result that the craft is often designed to pass the test (building unnecessary conservatism), rather than for flight. What is clear from this system engineering approach is the benefit of adoption of the correct boundary conditions (BC) in the design phase. Every time a clamped BC is introduced, whether it is at the spacecraft LVA interface or some other internal interface within the spacecraft (e.g. solar array etc) then the representativity of validation philosophy with respect to the true system response is compromised. As a consequence of artificial BCs it could be that failure modes are reordered and verification strategy does not encompass such true failure modes that would be developed as result of the correct interface representation or "soft mounting".

Besides the legal issues related to the Intellectual Properties of the various models, indeed it is well within the capabilities of current LV authorities to deliver a reduced/valid model of the LV and loads to SC Prime, and for these to use them to perform some SC/LV CLAs to drive the spacecraft design process. 
If we now draw a parallel between the SC/LV CLA situation and VST (see Figure 22), ideally the test house (which corresponds to the LV Authority for the CLA) has to develop reduced/valid model of their test equipment (which corresponds to the 'launcher'), and this could be delivered to the SC prime. As the SC prime is the central entity which on one side deals with the Launcher and on the other liaises with the test house, they are ideally positioned to ensure that whilst they design the spacecraft using Design CLA they also run VST to develop a suitable test verification campaign which minimizes over testing.

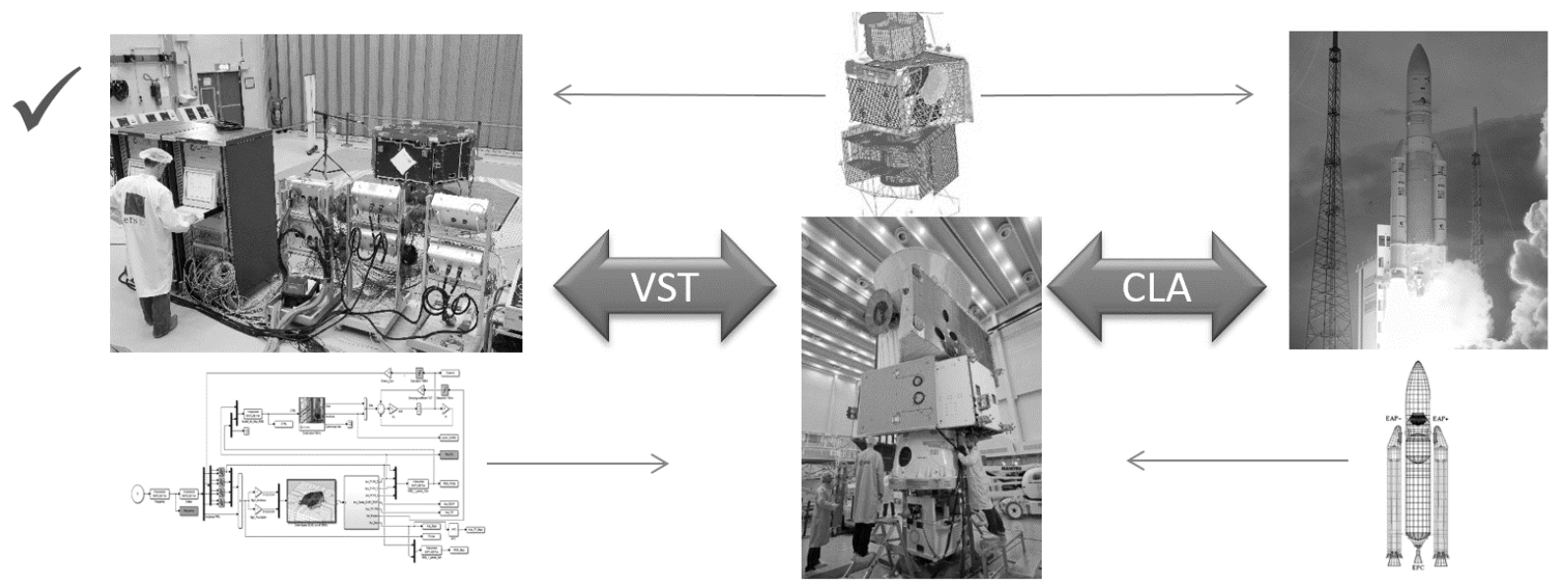

Figure 22: Virtual Shaker Testing and Coupled Load Analysis can be considered equivalent processes as in one case the satellite is coupled with the test equipment, whereas in the other it is coupled with the rocket.

The satellite is the common element in the two processes.

Alternatively the test houses could run the VST for the Satellite prime as pre-test activity, and use this to refine the parameters and profile to be used in the physical tests. However this scenario has the potential for a significant issue. If the first time that the physical satellite is actually put on the shaker a poor level of correlation is determined, it will be necessary to update the Satellite FEM, and this activity cannot be performed by the test house, as the FEM and related knowhow (which is necessary for the FEM update), resides with the prime. Hence the prime will have to perform the FEM update, and to carry out this exercise properly (removing the influence of the test equipment) the prime needs the capability to run the VST.

Some test houses currently do not have at their disposal validated models of their equipment, and indeed these will have to be developed, and then condensed or somehow packaged as black box models, in order to be delivered to SC primes without giving away any IP of the test house or test equipment suppliers. Clearly this will involve some cost for the test house which will be probably shared with the clients; however this will be a one-off cost that will be offset 
by the cost savings afforded by the VST procedure. Mainly, de-risking test activities, eliminating or at least reducing costly interruptions of test runs, and improving the quality of the correlation (which in turn allows to reduce the margins) ultimately improve the quality and cost of the final product.

\section{Conclusions}

In this article the motivations to carry out a VST procedure have been investigated in detail. Real examples of the influence on the test facilities equipment on test results have been examined, and it is concluded that ignoring these factors could have significant consequences on the test itself and the following FEM correlation/update process.

The various uses of VST as pre- and post- test tool have been proposed and applied in real scenarios using large satellite FEMs. This work shows that VST can give clear indications of frequency regions where the mismatch between FEM and test is due to inaccuracies of the FEM and other frequency regions where the mismatch is due to the test equipment.

The capability to model the effects of the test facility on the test results allows to improve the representativeness of the FEM as the correlation and FEM update processes can take these effects into account preventing an inconsistent correlation. Indeed carrying out VST adds some effort to the overall test and correlation activities which could impact cost and schedule, however its use during the test preparation activities, helping selecting test run parameters, or pilots locations, or helping to design the notch could prevent unpleasant surprises and costly delays during the physical tests. VST during the satellite FEM correlation process can help to identify issues related to the satellite FEM, and separate

those from the effects of the facility (shaker/slip table, controller), which otherwise could be included in the FEM of the satellite undermining its representativeness of the flight hardware. Whether these advantages justify the extra "analysis" effort involved in VST will depend on the specific cases, but it is expected that for large test items, where significant coupling with the facility is expected, the benefits of VST should justify its implementation.

This work also shows a new perspective, drawing parallels between VST and satellite/launch vehicle Coupled Load Analysis, and indicates a different philosophy to address the FEM validation process, which offers some technical advantages with respect to the current status quo. 


\section{References}

1. Allemang, R. J., and Brown, D. L. "A correlation coefficient for modal vector analysis," Proceedings of the 1st international modal analysis conference. SEM, Orlando, 1982.

2. Van der Auweraer, H., Iadevaia, M., Emborg, U., Gustavsson, M., Tengzelius, U., and Horlin, N. "Linking test and analysis results in the medium frequency range using principal field shapes," Proceedings of the international seminar on modal analysis. Vol. 2, Katholieke Universiteit Leuven, 1999, pp. 823-830.

3. Wijker, J. J. Mechanical vibrations in spacecraft design: Springer Science \& Business Media, 2004.

4. Nefske, D., and Sung, S. "Correlation of a coarse-mesh finite element model using structural system identification and a frequency response assurance criterion," Proceedings-SPIE the International Society for Optical Engineering. SPIE International Society for Optical Engineering, 1996, pp. 597-602.

5. Aglietti, G., Walker, S., and Kiley, A. "On the use of SEREP for satellite FEM validation," Engineering Computations Vol. 29, No. 6, 2012, pp. 580-595. doi: http://dx.doi.org/10.1108/02644401211246292

6. Sairajan, K., and Aglietti, G. "Study of Correlation Criteria for Base Excitation of Spacecraft Structure," AIAA Journal of Spacecraft and Rockets Vol. 51, No. 1, 2014, pp. 106-116. doi: 10.2514/1.A32457

7. Avitabile, P., Pechinsky, F., and O'Callahan, J. "Study of Modal Vector Correlation Using Various Techniques for Model Reduction," International Modal Analysis Conference (IMAC). Bethel, CT, 1992.

8. Warren, C., Niezrecki, C., Avitabile, P., and Pingle, P. "Comparison of FRF Measurements and Mode Shapes Determined Using Optically Image Based, Laser, and Accelerometer Measurements," Mechanical Systems and Signal Processing Vol. 25, No. 6, 2011, pp. 2191-2202. doi: 10.1016/j.ymssp.2011.01.018

9. "ECSS-E-ST-32-11 Modal Survey Assessment." European Cooperation for Space Standardization, ESA-ESTEC, Noordwijk, the Netherlands, 2008, p. 78.

10. $\quad$ "Load Analyses of Spacecraft and Payloads." NASA STD 5002, 1996, p. 20.

11. Friswell, M. I., and Mottershead, J. E. Finite Element Model Updating in Structural Dynamics: Kluwer Academic Publishers Group, 1995.

12. Mercer, J., Aglietti, G., Remedia, M., and Kiley, A. "Investigations Concerning the Effectiveness of the Spacecraft Finite Element Model Correlation and Update Process," 14th European Conference on Spacecraft Structures, Materials \& Environmental Testing (ECSSMET). Toulouse, France, 2016.

13. Sairajan, K., and Aglietti, G. "Robustness of System Equivalent Reduction Expansion Process on Spacecraft Structure Model Validation," AIAA Journal Vol. 50, No. 11, 2012, pp. 2376-2388.

14. Remedia, M., Aglietti, G., Appolloni, M., Cozzani, A., and Kiley, A. "Multi-DOF Transient Validation by Means of Virtual Testing," AIAA/ASCE/AHS/ASC Structures, Structural Dynamics, and Materials Conference, AIAA SciTech Forum. Kissimmee, Florida, 2018.

15. Sorge, R. "Space Power Facility-Capabilities for Space Environmental Testing Within a Single Facility." 2013.

16. Remedia, M., Aglietti, G., Appolloni, M., Cozzani, A., and Kiley, A. "An enhanced methodology for spacecraft correlation activity using virtual testing tools," Journal of Sound and Vibration Vol. 409, 2017, pp. 180-200.

17. Remedia, M., Aglietti, G., Appolloni, M., Cozzani, A., and Kiley, A. "Virtual Testing: A Pre- and Post-Test Tool for Base-Driven Spacecraft Testing," Aerospace Testing Seminar. Los Angeles, 2017.

18. Waimer, S., Manzato, S., Peeters, B., Wagner, M., and Guillaume, P. "Experimental and Analytical Approaches in a Virtual Shaker Testing Simulation Environment for Numerical Prediction of a Spacecraft Vibration Test," 36th IMAC, A Conference and Exposition on Structural Dynamics Vol. 8, Orlando, Florida, 2018.

19. Waimer, S., Manzato, S., Peeters, B., Wagner, M., and Guillaume, P. "Modelling and simulation of a closed-loop electrodynamic shaker and test structure model for spacecraft vibration testing," European Conference of Spacecraft Structures, Materials and Environmental Testing. Toulouse, France, 2016.

20. Xiang, S.-H., Liu, C., and Yan, T. "Virtual Vibration Test and Verification for the Satellite," 14th International Congress on Sound and Vibration. Cairns, Australia, 2007.

21. Liu, C., Xiang, S.-H., and Feng, Y.-Q. "Research on Virtual Vibration Test System for the Satellite," 17th International Congress on Sound and Vibration. Cairo, Egypt, 2010.

22. Ricci, B., Peeters, B., Fetter, R., Boland, D., and Debille, J. "Virtual shaker testing for predicting and improving vibration test performance," IMAC-XXVII. Society for Experimental Mechanics Inc., Orlando, Florida, US, 2009. 
23. Appolloni, M., and Cozzani, A. "Virtual testing simulation tool for the new QUAD Head Expander electrodynamic shaker," Environmental Testing of the Future - Reality in reliability, virtual testing \& trends in physical testing. ESA/ESTEC space centre Noordwijk, Holland, 2007.

24. Catalfamo, S., Smith, S., Morlock, F., Brake, M., Reuß, P., Schwingshackl, C., and Zhu, W. "Effects of Experimental Methods on the Measurements of a Nonlinear Structure," 34th IMAC, A Conference and Exposition on Structural Dynamics Orlando, Florida, 2016.

25. Appolloni, M., and Cozzani, A. "Virtual Testing for Vibration Tests: Methodology to Assess Facilities Performance and Modal Coupling," Aerospace Testing Seminar. Manhattan Beach, Los Angeles, CA, US, 2008.

26. Appolloni, M., and Cozzani, A. "Test predictions by an advanced numerical simulation tool for the Hydra Facility," Aerospace Testing Seminar. Los Angeles, USA, 2006.

27. Thoen, B., Cozzani, A., Appolloni, M., Salles, R., and Clark, A. "Controller upgrade for the HYDRA hydraulic shaker facility," 12th European Conference on Spacecraft Structures, Materials and Environmental Testing. ESTEC, the Netherlands, 2012.

28. Manzato, S., Bucciarelli, F., Arras, M., Coppotelli, G., Peeters, B., and Carrella, A. "Validation of a Virtual Shaker Testing approach for improving environmental testing performance," International Conference on Noise and Vibration Engineering (ISMA). Leuven, Belgium, 2014.

29. Hoffait, S., Marin, F., Simon, D., Peeters, B., and Golinval, J. "Virtual shaker testing at V2i: measured-based shaker model and industrial test case," International Conference on Noise and Vibration Engineering (ISMA). Leuven, Belgium, 2016.

30. Lang, G., and Synder, D. "Understanding the physics of electrodynamic shaker performance," Sound and Vibration Vol. 35, No. 10, 2001, pp. 24-33.

31. Nali, P., Di Pietro, V., Ladisa, P., Bitetti, G., Lumaca, F., and Bettacchioli, A. "Virtual Shaker Testing: actual achievements in TAS and future prospects," European Conference on Spacecraft Structures, Materials and Environmental Testing (ECSSMET). ESTEC, the Netherlands, 2018.

32. Allemang, R. J. "The Modal Assurance Criterion (MAC): Twenty Years of Use and Abuse," Journal of Sound and Vibration Vol. 37, No. 8, 2003.

33. Imregun, M., and Visser, W. J. A review of model updating techniques. Thousand Oaks, CA, ETATS-UNIS: Sage, 1991.

34. Mottershead, J. E., and Friswell, M. I. "Model Updating In Structural Dynamics: A Survey," Journal of Sound and Vibration Vol. 167, No. 2, 1993, pp. 347-375. doi: http://dx.doi.org/10.1006/jsvi.1993.1340

35. Levin, R. I., and Lieven, N. A. J. "Dynamic Finite Element Model Updating Using Simulated Annealing and Genetic Algorithms," Mechanical Systems and Signal Processing Vol. 12, No. 1, 1998, pp. 91-120. doi: http://dx.doi.org/10.1006/mssp.1996.0136

36. Boulkaibet, I., Mthembu, L., Marwala, T., Friswell, M. I., and Adhikari, S. "Finite element model updating using Hamiltonian Monte Carlo techniques," Inverse Problems in Science and Engineering, 2016, pp. 1-29. doi: 10.1080/17415977.2016.1215446

37. Papadimitriou, C., and Papadioti, D.-C. "Component mode synthesis techniques for finite element model updating," Computers \& Structures Vol. 126, 2013, pp. 15-28. doi: http://dx.doi.org/10.1016/j.compstruc.2012.10.018

38. Mercer, J., Aglietti, G., Remedia, M., and Kiley, A. "An assessment of spacecraft target mode selection methods," Acta Astronautica, 2017, pp. 537-553. 